\title{
"New is always better": Single center experience of over 200 cases of robotic single site cholecystectomy comparing the new da Vinci SP with the previous Si/Xi systems
}

\author{
Young-Dong YU* , Hye-Sung JO, Dong-Sik KIM
}

Department of Surgery, Korea University Anam Hospital, Seoul, Korea

Introduction: The da Vinci surgical system released its new pure single-port platform, the da Vinci SP, offering improvements and refinements such as articulating instruments for established robotic single-site procedures. We describe our institution's experience with robotic single site cholecystectomy utilizing the new da Vinci single-port (SP) robotic system and compare the results with the previous da Vinci Si/Xi system.

Methods: We reviewed the medical records and compared the perioperative outcomes of patients who underwent robotic single site cholecystectomy using the da Vinci SP model with the previous Si and Xi models.

Results: Of the 238 patients, there were 120 and 118 patients in the SP and Si/Xi group, respectively. The mean age of patients in the SP group was 48.7 years which was significantly higher than the Si/Xi group $(p=0.002)$. However, the BMI of patients in both groups was similar. In both groups, the most common indication for cholecystectomy was gallbladder stone. However there more patients diagnosed with acute cholecystitis in the SP group (4.2\%). The mean docking time ( $3.20 \pm 1.40 \mathrm{~min})$, console time (21.63 $\pm 13.34 \mathrm{~min})$ and total operative time $(48.42 \pm 18.33 \mathrm{~min})$ was significantly shorter in the SP group. However the conversion rate, complication rate and hospital stay was similar between the 2 groups.

Conclusions: Robotic single site cholecystectomy using da Vinci SP is safe and feasible. Due to its refinements, it requires less time for preparation and operation than the previous $\mathrm{Si}$ or Xi models and has showed promise to include more challenging indications such as acute cholecystitis. 